Journal of Case Reports 2020;10(4):241-243

\title{
Transient Hypotension after Submucosal Infiltration of Xylocaine with Adrenaline Solution during Functional Endoscopic Sinus Surgery
}

\author{
Vikas Sharma, Avaneesh Sandupatla, Sunil Dhole \\ Department of Cardiac Anesthesia, Fortis Escorts Heart Institute, New Delhi, India.
}

\section{Corresponding Author: \\ Dr Vikas Sharma \\ Email:drvikas661@gmail.com}

This is an Open Access article distributed under the terms of the Creative Commons Attribution License (creativecommons.org/ licenses/by/3.0).

Received

Accepted

Published

July 28,2020

September 3, 2020

November 25, 2020

\begin{abstract}
Introduction: Xylocaine or normal saline along with 1:2,00,000 adrenaline is commonly used for presurgical infiltration. Local infiltration containing adrenaline often causes cardiovascular side effects which may be variable ranging from tachycardia, hypertension, arrhythmias and hypotension in some cases. Case Report: We report a case of 54 year old female a known case of hypothyroidism, dyslipidemia with current admission diagnosis of deflected nasal septum, bilateral nasal polyposis and chronic sinusitis who underwent Functional Endoscopic Sinus Surgery (FESS) and septoplasty. Preprocedure the surgical site was infiltrated with 1:2,00,000 freshly prepared xylocaine with adrenaline solution. Subsequent to this infiltration patient developed hypotension which was transient in nature and responded to intravenous fluid bolus. Conclusion: The infiltration of xylocaine with adrenaline may lead to $\beta 2$ receptor activation leading to skeletal muscle vasodilation and decrease in preload leading to hypotension. This was the most probable attributable cause of transient hypotension in our case although this hypotensive effect is not seen commonly.
\end{abstract}

Keywords: Hypotension, Lidocaine, Nasal Septum, Saline Solution, Sinusitis.

\section{Introduction}

In patient undergoing Functional Endoscopic Sinus Surgery (FESS) and septoplasty, the nasal mucosa is often infiltrated with local anaesthetic solution that contains adrenaline to aid hemostasis. Local infiltration containing adrenaline, often cause cardiovascular side effects which may be variable ranging from tachycardia, hypertension, arrhythmias and hypotension in some cases. Transient hypotension following the infiltration of adrenaline containing local anaesthetics may be caused by stimulation of $\beta 2$ adrenoceptors in skeletal muscles by adrenaline producing vasodilation [1].

\section{Case Report}

A 54 years old female came with chief complaints of nasal blockade since last few months along with history of snoring and occasional headaches. After clinical evaluation and relevant investigations patient was diagnosed as a case of deflected nasal septum with bilateral nasal polyposis and chronic sinusitis. The co-morbidities were hypothyroidism with present euthyroid state and dyslipidemia. Pre-operatively she underwent exercise electrocardiogram which was mildly positive. Otherwise patient had no cardiac symptoms and had good exercise tolerance. Patient was planned for FESS along with removal of nasal polyps and correction of deflected nasal septum. 
General anaesthesia along with standard monitoring was planned for the procedure. During anaesthesia patient was monitored using 2 lead electrocardiogram (Lead II and V 5), pulse oximetry, temperature, capnography and anaesthesia gas monitoring. Invasive arterial blood pressure was monitored in lieu of pre-operative mildly positive electrocardiogram exercise testing and dyslipidemia. Right radial artery was cannulated with the 20 gauge flow switch cannula for invasive arterial pressure monitoring.

Anaesthesia was induced with propofol, fentanyl and atracurium in usual doses and maintained with oxygen air mixture, sevoflurane and intermittent muscle relaxation with atracurium. Paracetamol and diclofenac sodium was given to supplement analgesia along with fentanyl. All drugs were used in the standard doses. Before starting surgical procedure, nasal mucosa was infiltrated with $1: 2,00,000$ xylocaine with adrenaline solution (14 ml). Approximately 1-2 minutes after the injection of xylocaine with adrenaline patient developed hypotension; the mean blood pressure which was maintaining around $70-75 \mathrm{~mm}$ of $\mathrm{Hg}$ dropped down to $40-45 \mathrm{~mm}$ of $\mathrm{Hg}$ and this rapid change was easily visible on monitor as invasive arterial blood pressure was being monitored. This hypotensive episode was transient in nature and responded to fluid bolus in form of 200-300 ml of Ringer's Lactate solution and came to baseline levels in a few minutes.

During the further course of surgery the hemodynamic parameters remained stable. No further infiltrarion was given using xylocaine with $1: 2,00,000$ adrenaline solution. After the completion of surgical procedure muscle relaxation was reversed with neostigmine and glycopyrrolate; patient was extubated uneventfully and shifted to post-operative area. The patient did well postoperatively and was discharged on the $2^{\text {nd }}$ postoperative day.

\section{Discussion}

Local anaesthetics infiltration containing adrenaline, which often cause cardiovascular side effects are routinely used in functional endoscopic sinus surgery for the main purpose of hemostasis. This infiltration also allows better visualization of vascular structures during surgery [2]. Head and neck is a highly vascular area and absorption of adrenaline is rapid. Submucosal infiltration in the nasal mucosa of solutions containing various concentrations of epinephrine may lead to variable hemodynamic changes. Transient hypertension (lasting 1-5 $\mathrm{min}$ ), and arrhythmias may result due to inadvertent high concentration $(10-20 \mu \mathrm{g} / \mathrm{mL})$, large volume of solutions, rapid infiltration or accidental intravenous infiltration. Numerous reports have shown that injection of adrenaline, even in therapeutic doses, can lead to increased heart rate and stroke volume, resulting in arrhythmias in susceptible patients $[3,4]$. The incidence of cardiovascular toxic adverse effects has been shown to increase in a dose-dependent manner. Sometimes dilute adrenaline solutions may cause fall in blood pressure due to activation of $\beta 2$ receptors in skeletal muscle. Prolonged intra-operative hypotension after subcutaneous infiltration of diluted adrenaline is an uncommon scenario but a possibility as reported by Shyam Bhandari et al. [5].

Adrenaline is really a sympathomimetic amine with each alpha as well as beta-adrenergic receptor agonist effects. Skin, mucosa, as well as kidney arterioles display vasoconstriction on account of alpha-receptor predominant activation. Low concentrations of adrenaline lead to preferential $\beta 2$ receptor activation leading to vasodilatation in bronchiolar smooth muscle while increased ranges activates alpha-receptormediated vasoconstriction in vascular smooth muscle [6]. Due to high vascularity of the nasal area even dilute adrenaline solutions $(1: 2,00,000)$ may cause fall in blood pressure due to activation 
of $\beta 2$ receptors in the skeletal muscles. Altered pharmacokinetics and pharmacodynamics of drug can lead to exaggeration of some of the effects. The activation of $\beta 2$ receptors may be further exaggerated if the patient is already anaesthetized as there is blunting of sympathetic response. In one of the studies it was mentioned that the lighter plane of anaesthesia may be protective against adrenaline induced hypotension [7]. The increased $\beta 2$ receptor sensitivity may be one of the reasons for profound hypotension. Another remote possibility may be the occurrence of Bezold-Jarisch reflex which is a cardioprotective reflex [8].

\section{Conclusion}

The hypotensive effects of infiltration of epinephrine containing solutions at subtherapeutic concentrations are attributed to the preferential stimulation of the $\beta 2$ receptors at lower concentrations. This was the most probable attributable cause of transient hypotension in our case although this hypotensive effect is not seen commonly. The controversies concerning hemodynamic effects of adrenaline in local infiltration are widely discussed, but there is no definite conclusion. Close hemodynamic monitoring should always be considered, whenever adrenaline containing solutions are used for presurgical infiltration for head and neck surgeries. We should remain vigilant whenever these techniques are used in conjunction with adrenaline infiltration as precipitous fall in blood pressure may occur.

Contributors: VS: Manuscript writing, and literature review; AS: manuscript editing, and references. SD: critical inputs into the manuscript. VS will act as a study guarantor. All authors approved the final version of this manuscript and are responsible for all aspects of the study.

Funding: None; Competing interests: None stated.

\section{References}

1. Wilis Mathew D, Sanders M, Sinha S, Mirza S, Andrzejowsi JC. The pathophysiology of early hypotension following epinephrine-containing local anesthetic infiltration of the nasal mucosa in patients undergoing endoscopic transsphenoidal hypophysectomy: A prospective, observational study. Neurosurg Anaesthesiol. 2017;29:330;34.

2. Yang JJ, Wang QP, Wang TY, Sun J, Wang, Zuo D Xu JG. Marked hypotension induced by adrenaline contained in local anesthetic. The Laryngoscope. 2005;115:348-352.

3. Horrigan RW, Eger EI, Wilson C. Epinephrine-induced arrhythmias during enflurane anaesthesia in man: a nonlinear dose-response relationship and dose dependent protection from lidocaine. Anesthesia Analgesia. 1978;57:547-550.

4. Murthy HS, Rao GS. Cardiovascular responses to scalp infiltration with different concentrations of epinephrine with or without lidocaine during craniotomy. Anesth Analges. 2001;92:1516-1519.

5. Shyam B, Mazamil S, Bano S, Siddiqui SS, Ahmad. A case of hypotension after intranasal adrenaline infiltration causing a clinical dilemma during the intraoperative period. Indian J Anaesthes. 2011;55:74-78.

6. Li WY, Zohu ZQ, Ji JF, Li ZQ, Yang JJ, Shang RJ. Relatively light general anaesthesia is more effective than fluid expansion in reducing the severity of epinephrine induced hypotension during FESS. Chin Med J. 2007;120:1299-1302.

7. Sun LS, Schwarzenberger JC. Cardiac Physiology. In: Miller RD, (editor) Miller anaesthesia. Philadelphia: Churchill Livingstone. 2010;7:409.

8. Yang JJ, Li WY, Jil Q, Wang ZY, Sun J, Wang QP, et al. Local anesthesia for functional endoscopic sinus surgery employing small volumes of epinephrine-containing solutions of lidocaine produces profound hypotension. Acta Anaesthesiol Scand. 2005;49:1471-1476. 